\title{
Upper Bounds on Geometric Permutations for Convex Sets
}

\author{
Rephael Wenger \\ School of Computer Science, McGill University, 805 Sherbrooke Street West, \\ Montreal, Quebec H3A 2K6, Canada
}

\begin{abstract}
Let $A$ be a family of $n$ pairwise disjoint compact convex sets in $R^{d}$. Let $\Phi_{d}(m)=2 \sum_{i=0}^{d-1}\left(\begin{array}{c}m-1 \\ i\end{array}\right)$. We show that the directed lines in $R^{d}, d \geq 3$, can be partitioned into $\Phi_{d}\left(\left(\begin{array}{l}n \\ 2\end{array}\right)\right)$ sets such that any two directed lines in the same set which intersect any $A^{\prime} \subseteq A$ generate the same ordering on $A^{\prime}$. The directed lines in $R^{2}$ can be partitioned into $12 n$ such sets. This bounds the number of geometric permutations on $A$ by $\frac{\varepsilon}{2} \Phi_{d}\left(\left(\begin{array}{l}n \\ 2\end{array}\right)\right)$ for $d \geq 3$ and by $6 n$ for $d=2$.
\end{abstract}

\section{Introduction}

Let $A=\left\{a_{1}, a_{2}, \ldots, a_{n}\right\}$ be a family of $n$ pairwise disjoint compact convex sets in $R^{d}$. A common transversal for $A$ is a line intersecting each set in $A$. Each common transversal intersects the sets in $A$ in a unique order, up to reversal. Katchalski, Lewis, Zaks, and Liu called this ordering and its "reversal" a geometric permutation of $A[\mathrm{KLZ}],[\mathrm{KLL1}]$. They showed that any family $\boldsymbol{A}$ of $\boldsymbol{n}$ disjoint compact convex sets in $R^{2}$ has at most $\left(\begin{array}{l}n \\ 2\end{array}\right)$ geometric permutations.

Geometric permutations partition the common transversals of $A$ into disjoint sets such that any two transversals in the same set intersect $A$ in the same order. We can extend the idea of geometric permutations to include orderings from lines which intersect a subset of $A$. Two lines which intersect $A^{\prime} \subseteq A$ can be associated if they intersect $A^{\prime}$ in the same order. We wish to partition the directed lines in $R^{d}$ into as few sets as possible such that every two lines in the same set which intersect any $A^{\prime} \subseteq A$ generate the same ordering on $A^{\prime}$. Each geometric permutation is associated with a unique pair of sets from the partition. Thus bounding the size of the partition bounds the number of geometric permutations. 
We define a new term, a separation set for $A$. We say that a hyperplane $h$ separates $a_{i} \in A$ from $a_{j} \in A$ if $a_{i}$ lies in one of the closed half-spaces bounded by $h$ and $a_{j}$ lies in the other. We say that a hyperplane $h$ strictly separates a convex set $a_{i}$ from a convex set $a_{j}$, if $h$ separates $a_{i}$ from $a_{j}$ and $h \cap a_{i}=h \cap a_{j}=\varnothing$. If $H$ is a set of hyperplanes in $R^{d}$, then we say that $H$ is a separation set for $A$ if, for every pair of elements, $a_{i}, a_{j} \in A$, there exists a hyperplane $h \in H$ such that $h$ strictly separates $a_{i}$ from $a_{j}$. Obviously, there exist infinitely many different sets which are separation sets for $A$.

Let $H$ be a finite set of hyperplanes in $R^{d}$ and let $|H|$ be the number of elements in $H$. Define

$$
\Phi_{d}(m)=2 \sum_{i=0}^{d-1}\left(\begin{array}{c}
m-1 \\
i
\end{array}\right)
$$

and define $\Psi_{d}(H)$ to be the number of cones in the pencil obtained by translating the hyperplanes in $H$ to the origin. Winder [W] proved that $\Psi_{d}(H) \leq \Phi_{d}(|H|)$.

We present three main theorems in this paper. Bill Lenhart provided the inspiration for Theorem 3 .

Theorem 1. Let $A$ be a family of pairwise disjoint compact convex sets in $R^{d}$ and $H$ be some separation set for $A$. The directed lines in $R^{d}$ can be partitioned into $\Psi_{d}(H)$ sets such that every two directed lines in the same set which intersect any $A^{\prime} \subseteq A$ generate the same ordering on $A^{\prime}$.

Theorem 2. If $A$ is a family of $n$ pairwise disjoint convex polygons in $R^{2}$, then there exists a separation set $L$ for $A$ where each line in $L$ is parallel to some edge of a polygon in $A$.

Theorem 3. Given $A$, a family of $n$ pairwise disjoint compact convex sets in $R^{2}$, there exists a family $B$ of $n$ pairwise disjoint compact convex polygons, such that:

(i) each convex set in $A$ is entirely contained in a unique polygon in $B$,

(ii) the total number of edges in all the polygons in $B$ is at most $12 n$, and

(iii) if $L$ is the set of lines containing the edges of the polygons in $B$, then $\Psi_{2}(L) \leq 12 n$.

For every pair of disjoint compact convex sets, there exists some hyperplane which strictly separates the pair [G]. By choosing such a hyperplane for every pair, we can always construct a separation set $H$ for $n$ compact convex sets containing $\left(\begin{array}{l}n \\ 2\end{array}\right)$ elements. Since $\Psi_{d}(H) \leq \Phi_{d}(|H|)$, we have the following corollary to Theorem 1:

Corollary 1. If $A$ is a family of $n$ pairwise disjoint compact convex sets in $R^{d}$, the directed lines in $R^{d}$ can be partitioned into $\Phi_{d}\left(\left(\begin{array}{l}n \\ 2\end{array}\right)\right)$ sets such that any two directed lines in the same set which intersect any $A^{\prime} \subseteq A$ generate the same ordering on $A^{\prime}$. 
Finally, combining Theorems 2 and 3, we can always find a separation set $L$ for $n$ convex sets in $R^{2}$ such that $\Psi_{2}(L) \leq 12 n$. Applying Theorem 1 , we have the following corollary:

Corollary 2. If $A$ is a family of n pairwise disjoint compact convex sets in $R^{2}$, the directed lines in $R^{2}$ can be partitioned into $12 n$ sets such that any two directed lines in the same set which intersect any $A^{\prime} \subseteq A$ generate the same ordering on $A^{\prime}$.

As noted before, each geometric permutation is associated with a unique pair of sets in the partition of directed lines. Thus the number of geometric permutations on $A$ is bounded by half the size of the partition. Corollaries 1 and 2 imply that there are at most $\frac{1}{2} \Phi_{d}\left(\left(\begin{array}{l}n \\ 2\end{array}\right)\right)$ geometric permutations of $A$ in $R^{d}$ and at most $6 n$ geometric permutations of $A$ in $R^{2}$.

\section{Upper Bounds on the Number of Geometric Permutations}

We now prove Theorem 1 . Let $A=\left\{a_{1}, a_{2}, \ldots, a_{n}\right\}$ be a family of $n$ pairwise disjoint compact convex sets in $R^{d}$ and let $H=\left\{h_{1}, h_{2}, \ldots, h_{m}\right\}$ be a separation set of $A$ containing $m$ elements. Let $\left\{u_{1}, u_{2}, \ldots, u_{m}\right\}$ be a set of normal vectors to $H$ where $u_{k}$ is normal to $h_{k}$.

Let $v$ be a vector in $R^{d}$ and let $T$ be the set of all lines with direction $v$. Let $h_{k}$ be the hyperplane separating $a_{i}$ and $a_{j}$ and assume $\left(a_{j}-a_{i}\right) \cdot u_{k}>0$. If $v \cdot u_{k}>0$, then any line in $T$ must intersect $a_{i}$ before $a_{j}$. If $v \cdot u_{k}<0$, then any line in $T$ must intersect $a_{j}$ before $a_{i}$. Furthermore, even if no line with direction $v$ intersects $a_{i}$ and $a_{j}$ simultaneously, $\operatorname{sgn}\left(v \cdot u_{k}\right)$ still determines an ordering relation on $a_{i}$ and $a_{j}$. Thus $\operatorname{sgn}\left(v \cdot u_{k}\right), k=1, \ldots, m$, determines the relative order in which any line with direction $v$ intersects any two elements of $A$.

Consider the cones created by hyperplanes through the origin with normals $u_{k}, k=1, \ldots, m$. The values of $\operatorname{sgn}\left(v \cdot u_{k}\right), k=1, \ldots, m$, are determined by the cone into which $v$ points. Any two lines which point into a given cone and intersect some $A^{\prime} \subseteq A$ generate the same ordering on $A^{\prime}$. There are at most $\Psi_{d}(H)$ cones, and so the directed lines can be partitioned into $\Psi_{d}(H)$ sets with the desired property. This proves Theorem 1.

\section{Separation Sets for Convex Polygons}

Let $A$ be a family of pairwise disjoint compact convex polygons in $R^{2}$. For Theorem 2, we show how to construct a separation set $L$ for $A$ where each line in $L$ is parallel to some edge in $A$. We need merely show that for any two polygons, $a_{i}$ and $a_{j}$, there exists some line strictly separating $a_{i}$ from $a_{j}$ and parallel to some edge in $A$.

Let $l^{*}$ be some line strictly separating convex polygon $a_{i}$ from convex polygon $a_{j}$ and tangent to $a_{i}$ at some vertex. Let $l_{1}$ and $l_{2}$ be the two lines containing the 


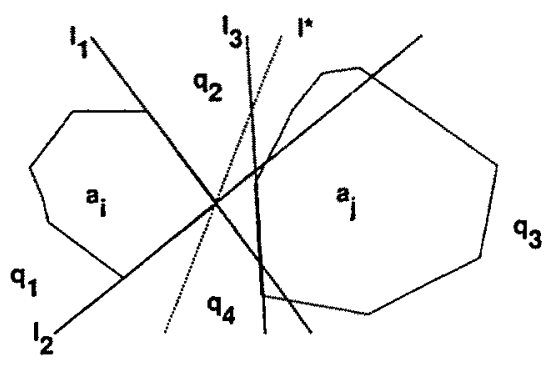

Fig. 1. Convex polygons $a_{\text {, and }} a_{r}$.

edges of $a_{i}$ which meet at that vertex. $l_{1}$ and $l_{2}$ divide the plane into four quadrants, $q_{1}, q_{2}, q_{3}, q_{4}$, with $a_{i}$ lying wholly in one quadrant, say $q_{1}$ (see Fig. 1). We include in each quadrant the boundary of that quadrant.

Since $l^{*}$ separates $a_{j}$ from $a_{i}, a_{j}$ does not intersect quadrant $q_{1}$. If $a_{j}$ does not intersect quadrant $q_{2}$, then $l_{2}$ separates $a_{i}$ from $a_{j}$. If $a_{j}$ does not intersect quadrant $q_{4}$, then $l_{1}$ separates $a_{i}$ from $a_{j}$. By translating either of these separators slightly toward $a_{j}$, we have a line strictly separating $a_{i}$ from $a_{j}$ and parallel to a line through an edge of $a_{i}$.

Assume $a_{j}$ contains a point from quadrant $q_{2}$ and from quadrant $q_{4}$. Some edge of $a_{j}$ must intersect $l_{1}$. Let $l_{3}$ be the line containing that edge. $l_{3}$ separates $a_{1}$ from $a_{2}$ so by translating it slightly toward $a_{j}$ we have a line strictly separating $a_{i}$ from $a_{j}$ and parallel to an edge of $a_{i}$.

For each pair of polygons $a_{i}, a_{j} \in A$, we add to $L$ a line strictly separating $a_{i}$ from $a_{j}$ and parallel to some edge in $a_{i}$ or $a_{j} . L$ is a separation set for $A$ such that every line in $L$ is parallel to some edge of a polygon in $A$, fulfilling the conditions in Theorem 2.

\section{Embedding Compact Convex Sets in Polygons}

Given $n$ pairwise disjoint compact convex sets, we show how to embed these convex sets in $n$ pairwise disjoint compact convex polygons satisfying the conditions in Theorem 3 . We first note that $n$ pairwise disjoint convex sets can be embedded in $n$ pairwise disjoint convex polygons using a total of not more than $n(n+2)$ edges. Choose some convex set $a$ and find $n-1$ lines separating $a$ from the $n-1$ other convex sets. These $n-1$ lines bound $n-1$ half-planes containing $a$. If the intersection of these half-planes is unbounded, we can add three suitable half-planes containing $a$ such that the intersection of the $n+2$ half-planes is bounded. The intersection of these half-planes forms a polygon with at most $n+2$ edges which contains $a$. Repeat the procedure $n$ times to get a set $A$ of $n$ pairwise disjoint convex polygons containing the $n$ convex sets.

Following [PS], a complete triangulation of a finite set of points $S$ is a planar graph whose vertices are the points of $S$, whose edges are nonintersecting line segments joining the points in $S$, and whose faces (including the external face) are bounded by triangles. We define a new type of triangulation, a complete 


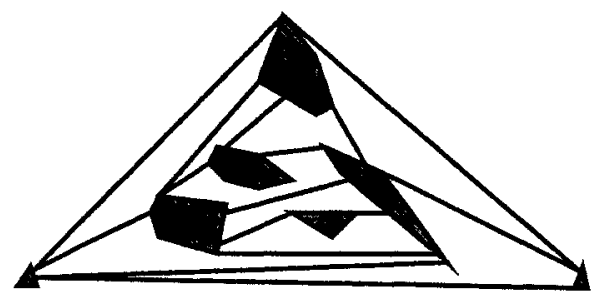

Fig. 2. A complete triangulation of convex polygons.

triangulation of a finite family of convex polygons $A$ is a planar graph whose vertices can be mapped to polygons in $A$, whose edges can be mapped to nonintersecting line segments joining two polygons and not intersecting any other polygon, and whose faces (including the external face) are bounded by triangles (see Fig. 2). We allow graphs to have multiple edges, i.e., more than one edge between any two vertices.

Let $c$ be some polygon in $A$ which lies on the convex hull of $A$. Add two small triangles, $d$ and $d^{\prime}$, to $A$ such that the convex hull of $A^{\prime}=A \cup\left\{d, d^{\prime}\right\}$ is three line segments between $c, d$, and $d^{\prime}$. The convex hull of $A^{\prime}$ minus the polygons in $A^{\prime}$ forms a polygon with holes. Triangulate this polygon with holes [PS]. This triangulation induces a planar graph on the polygons in $A^{\prime}$ where polygons are mapped to vertices and triangulation line segments to edges. The faces in this planar graph are bounded by two or three edges. If a face is bounded by only two edges, one of the bounding edges and its corresponding line segment is redundant. Remove these redundant edges and line segments until all faces are bounded by three edges. The resultant planar graph $G$ is a complete triangulation of $A^{\prime}$.

Given this triangulation $G$ of $A^{\prime}$ we can embed the $n$ pairwise disjoint convex polygons in $A$ into $n$ pairwise disjoint convex polygons with at most $12 n$ edges. For each triangulation line segment $s$ connecting $a, b \in A^{\prime}$, let $l_{s}$ be the line containing $s$. Let $l_{a, b}$ be some line strictly separating $a$ from $b$.

For each convex set $a \in A^{\prime}$, let $N(a)$ be a list of the neighbors of $a$. Let $F(a)$ be a list of the faces which have $a$ as a vertex. Let $L_{1}(a)=\left\{l_{a, b}: b \in N(a)\right\}$ and let $L_{2}(a)=\left\{l_{s}: s\right.$ lies on a face of $F(a)$ and connects $\left.b, b^{\prime} \in N(a)\right\}$. Let $L_{0}(a)$ be the union of the lines in $L_{1}(a)$ and $L_{2}(a)$ slightly translated toward $a$. Let $P(a)$ be the intersection of the half-planes containing $a$ and bounded by the lines in $L_{0}(a)$. For any $a \in A$ other than $c, P(a)$ is bounded by the cycle of the neighbors of $a$ and the triangulation line segments between them (see Fig. 3). Thus $P(a)$ is a convex polygon containing $a$. By a judicious choice of $l_{c, d}$ and $l_{c, d^{\prime}}, P(c)$ is also a convex polygon containing $c$. We claim that $B=\{P(a): a \in A\}$ is a family of $n$ pairwise disjoint compact convex polygons such that each $a \in A$ is contained in $P(a)$ and the total number of edges in the polygons in $B$ is $12 n$.

Assume $a, b \in A$ are neighbors in $G . P(a)$ and $P(b)$ are both bounded by a translation of the same separator, $l_{a, b}$ of $a$ and $b$. Since $P(a)$ and $P(b)$ are in different half-planes bounded by $l_{a, b}, l_{a, b}$ must separate $P(a)$ from $P(b)$ and $P(a)$ and $P(b)$ are pairwise disjoint. 


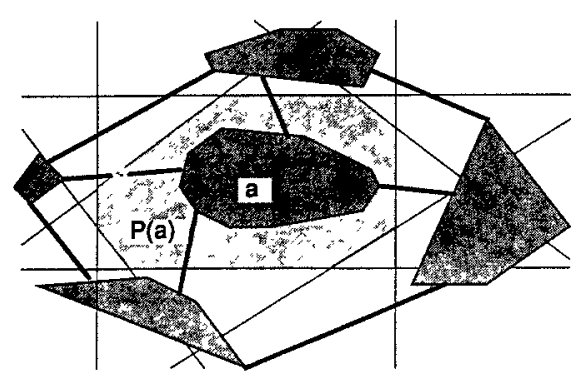

Fig. 3. Cycle of neighbors of $a$.

Now, assume $a, b \in A$ are not neighbors in $G . P(a)$ is entirely contained in a cycle of the neighbors of $a$ while $P(b)$ is entirely contained in a cycle of the neighbors of $b$. Since $b$ is not a neighbor of $a$, these two cycles must contain different regions in the plane and so $P(a)$ and $P(b)$ must be pairwise disjoint.

Let $m$ be the number of edges in $G$. $G$ is a planar graph in which every face is a triangle and there are $n+2$ vertices in $G$, so $m=3 n[\mathrm{H}]$. Each edge connects two neighbors, so the total number of neighbors over all vertices in the graph is $6 n$. The total number of edges in the polygons in $B$ is at most the total number of lines in $L_{0}(a)$ over all $a \in A$ :

$$
\sum_{a \in A}\left|L_{0}(a)\right| \leq \sum_{a \in A^{\prime}}\left|L_{0}(a)\right|=\sum_{a \in A}\left|L_{1}(a)\right|+\sum_{a \in A}\left|L_{2}(a)\right| \leq 6 n+6 n=12 n
$$

Therefore, the total number of edges in the polygons in $B$ is at most $12 n$.

Let $L$ be the set of lines containing the edges of the polygons in $B$. $L$ is a subset of $L^{\prime}=\bigcup_{a \in A^{\prime}} L_{0}(a)$. Each line in $L_{0}(a)$ is parallel to some line in some $L_{0}(b), a, b \in A^{\prime}$. Since the total number of lines in $L^{\prime}$ is at most $12 n, \Psi_{2}\left(L^{\prime}\right) \leq 12 n$ and so $\Psi_{2}(L) \leq 12 n$.

\section{Conclusion}

Katchalski et al. [KLZ] asserted that, for every $d$, there exists a constant $k_{d}$ and a family $A$ of $n$ pairwise disjoint compact convex sets in $R^{d}$ such that there are at least $k_{d} n^{d-1}$ geometric permutations of $A$. Corollary 1 implies an upper bound of $O\left(n^{2 d-2}\right)$ for the number of geometric permutations of $A$, leaving a wide gap for improvement. Villanger showed that for any $n$ there exist families of $n$ line segments in $R^{3}$ in which any hyperplane separates at most one line segment from one other line segment [T]. Thus, there exist a family $A$ of $n$ compact convex sets in $R^{d}$, for any $d \geq 3$, such that any separation set of $A$ must have $\left(\begin{array}{l}n \\ 2\end{array}\right)$ elements. In fact, by embedding Villanger's line segments in rectangular prisms, we see that Theorem 2 does not generalize to two dimensions. Reduction of the upper bounds must come from other directions. 
Much attention has been paid to geometric permutations of families of translates [K], [KLL1], [KLL2]. Are there separation theorems for families of translates? These theorems may increase our understanding about geometric permutations of families of translates.

We are also interested in reducing the $12 n$ edges needed for $n$ pairwise disjoint compact convex polygons to contain $n$ pairwise disjoint compact convex sets in $R^{2}$. Approximately $6 n$ edges are needed for any $n$ pairwise disjoint convex polygons containing $n$ hexagons in a lattice of hexagons. We do not know if any set of $n$ compact convex sets in $R^{2}$ can be contained in $n$ compact convex polygons in $R^{2}$ with a total of about $6 n$ edges. Finally, we do not know if Theorem 3 generalizes to three dimensions. Can any set of $n$ pairwise disjoint compact convex sets in $R^{3}$ be embedded in $n$ pairwise disjoint convex polyhedra such that the total number of faces is linear in $n$ ?

\section{Acknowledgments}

I would like to thank Bill Lenhart who provided the inspiration for Theorem 3 . I would also like to thank David Avis, Herbert Edelsbrunner, and Meier Katchalski, who helped in various aspects of writing this paper. Finally, I would like to thank the referees for helping to simplify the proofs in this paper.

\section{References}

[G] B. Grunbaum, Convex Polytopes (Wiley, New York, 1967).

[H] F. Harary, Graph Theory (Addison-Wesley, Reading, Massachusetts, 1969).

[K] M. Katchalski. On a Conjecture of Grunbaum on Common Transversals, to appear in Mathematica Scandinavica.

[KLL1] M. Katchalski, T. Lewis, and A. Liu, Geometric Permutations and Common Transversals, Discrete and Computational Geometry, 1 (1986), 371-377.

[KLL2] M. Katchalski, T. Lewis, and A. Liu, Geometric Permutations of Disjoint Translates of Convex Sets, to appear in Discrete Mathematics.

[KLZ] M. Katchalski, T. Lewis, and J. Zaks, Geometric Permutations for Convex Sets, Discrete Mathematics, 54 (1985), 271-284.

[PS] F. P. Preparata and M. I. Shamos, Computational Geometry (Springer*Verlag, New York, 1985).

[T] H. Tverberg, A Separation Property of Plane Convex Sets, Mathematica Scandinavica, 45 (1979), 255-260.

[W] R. O. Winder, Partitions of N-Space by Hyperplanes, SIAM Journal on Applied Mathematics, 14 (1966), 811-818.

Received November 14, 1986, and in revised form Seprember 28, 1987. 